occurring in low temperature fuel cells: anodic oxidation of molecular hydrogen, oxygen layers on electrodes and inhibition of fuel oxidations, the adsorption of carbonaceous species on platinum metals, the anodic oxidation of fuels, oxygen electrodes and finally corrosion of electrodes. The book then moves away from low temperature fuel cells and the next chapter deals with processes in fuel cells with molten carbonate electrolytes. Chapter fourteen describes processes in fuel cells with solid electrolytes. It is a great pity that in view of the relative promise of this type of fuel cell compared with low temperature systems, only seven pages are spent on it. The last two chapters deal with properties and models of porous electrodes respectively.

The author is well known for his work in the field and the book is authoritatively and well written. By current standards it is also reasonably priced. The book will be useful for experienced electrochemists who are looking for the most up to date (end of 1967) summary of the literature dealing with fundamental aspects of fuel cell research. It is unfortunately not possible to say that this book fills substantially a gap in the literature. F. GOODRIDGE

\section{PHYSICS EXPLAINED}

Atomic Physics

By E. V. Shpol'skii. Translated by A. Tybulewicz. English translation edited by $\mathrm{S}$. Chomet. Pp. 644. (Iliffe: London, July 1969.) $80 s$.

THIs book represents an ambitious attempt to provide a comprehensive introduction to atomic (as opposed to nuclear) physics. It begins with a description of the properties of the electron and their experimental determination, and it ends with the solution of the Schrödinger equation for the hydrogen atom. On the way, the fundamental measurements are described with far more care and attention to experimental factors than are common in an introductory text, and the theoretical background is developed in easy stages.

It is sometimes asked whether comprehensive books of this type covering so wide a range of topics can really be successful. The answer is that such books can be, but rarely are, successful; the present example undoubtedly comes into this rare category, so much so that it invites comparison with the finest of all examples of this genre in the field of atomic physics, namely, Atomic Physics, by Max Born. This last, of course, bears the stamp of a great physicist who made profound contributions to the subject he was writing about, and Shpol'skii's book does not quite match Born's in this respect. But he does emerge as an author with a deep understanding both of the subject matter itself and of the problems faced by a teacher. The student is steered with great skill through the complex interrelationship of theory and experiment in chapters devoted to atomic models, black body radiation, atomic spectra and the wave nature of matter. One has only to look at the excellent account of Bohr's theory of the hydrogen atom in chapter eight, and compare it with the almost superstitious versions often encountered in elementary texts, to confirm that here is an author in full command of the subtleties of physical meaning embedded in quantum theory. Likewise, there is an excellent account of Einstein's derivation of Planck's law, although for some reason the author fails to follow Einstein in using Wien's law to demonstrate by the correspondence principle that $E_{2}-E_{1}=h_{v}$.

Not surprisingly in a book of this length, I have minor eriticisms. The author occasionally makes rather heavy going of relatively elementary matters, as, for example, in the treatment of conservation of energy in classical mechanics in section 41, page 154. After very carefully and correctly developing the complex Fourier expansion of a real function in section 48 , complex numbers are misused in an important application in section 73 , where an essentially real function is equated to a complex function. (This error is almost universal in elementary texts. Born, needless to say, gets it right.) But these are small blemishes, and their significance should not be exaggerated. The one really surprising feature is that no mention is made of electron spin. This does seem to me to be a very serious omission, even from a deliberately elementary text, more particularly when twelve pages are devoted to such a non-elementary topic as the Mössbauer effect.

This book can be confidently recommended, with only the reservation just mentioned, for physics, and other science students beginning their university courses. They should emerge at the end of its 600 pages with important insights into some of the most fundamental features of the physical world.

W. R. Hindmarsh

\section{KINETIC THEORY}

\section{Kinetic Processes in Gases and Plasmas}

Edited by A. R. Hochstim. (Reentry Physics Series.) Pp. xiv +458 . (Academic Press: London and New York, May 1969.) $210 s$.

THrs volume forms one of a series concermed with "reentry physics", but is concerned with fundamental prob. lems of kinetic theory of far wider interest than this context would suggest. It takes the form of a collection of ten largely self-contained articles from twelve authors and, as is perhaps inevitable in such a collection, the level and indeed the apparent objectives of the presentation vary noticeably from one article to another.

The first five articles consist of systematic exposition of the equations of kinetic theory for both neutral gases and plasmas. There is considerable overlap, but the resulting gain in clarity, because each article illuminates different aspects of the derivations, more than compensates for this. The short article by M. B. Lewis on the inter-relationships between the Liouville, Boltzmann and Fokker-Planck equations is specially clear and precise (in spite of a slight confusion of notation on page 119). These relationships and others between these and the many other kinetic equations derived by various authors for special situations are well displayed in charts which illustrate three of the articles. These are extremely valuable.

The sixth article, by Hochstim and G. A. Massel, is by far the longest and is the least satisfactory. It deals in great detail with the application of kinetic theory to the calculation of transport coefficients in ionized gases. It is written in a style perhaps appropriate to a reference handbook but with, for example, frequent use of a notation "to be introduced and tabulated later" which must make its use for reference purposes extremely cumbersome. The authors make much of having extended the solution to the transport equations in series of Laguerre polynomials up to twenty terms (involving a $20 \times 20$ matrix which they tabulate in detail), but it is hard to find any attempt to justify such a tour de force.

The remaining four articles deal with chemical reactions considered as collision processes, reaction cross-sections, triple collisions, and methods of calculating inelastic collision cross-sections. All these are clear and helpful, but the two articles by F. T. Smith are outstanding for their clarity and precision of approach.

This is altogether a valuable and welcome book which will be useful to workers outside the immediate field as an excellent summary of current knowledge. The clear statements of the limitations of the various approximations and the careful distinction between fundamentally quantum mechanical features and those which can be understood classically are particularly welcome features. It is attractively and clearly produced and represents excellent value. I have found no errors apart from that in the notation mentioned earlier. M. G. RUSBRIDGE 